\title{
NEIGHBORHOOD CONTRACTIBLE SPACES
}

\author{
JOHN R. MARTIN ${ }^{1}$
}

\begin{abstract}
In this paper the concept of a neighborhood contractible space (or NC-space) is introduced. The class of NC-spaces is a proper subclass of the class of locally contractible compacta and it is shown that several classes of spaces, including the class of ANR-spaces, belong to the class of NC-spaces.
\end{abstract}

1. Introduction. It is well known that every ANR-space is locally contractible [1,p. 101] and that a finite-dimensional compactum is an ANR-space iff it is locally contractible [1, p. 122]. However, an elegant example due to Borsuk [1, p. 126] shows that even a contractible and locally contractible infinite-dimensional compactum need not be an ANR-space. It is Borsuk's example that provides the motivation for the definition of neighborhood contractible compacta since every neighborhood contractible compactum is locally contractible. but Borsuk's example fails to be neighborhood contractible. The purpose of this paper is to show that all ANR-spaces, AAR-spaces and AFS-spaces (see $[3,4]$ ) are neighborhood contractible spaces.

2. Notation and preliminaries. Apart from the notions of the cap over a continuum, absolute fixed point sets (AFS-spaces) and absolute approximate retracts ( $A A R$ spaces), the terminology used in this paper may be found in [1]. In particular, Hilbert space will be denoted by $E^{\omega}$ and the diameter of a set $M$ will be denoted by $\delta(M)$. Although definitions for the first three concepts may be found in $[3,4]$, we include them in this section for the sake of completeness.

Let $C$ be a continuum lying in $E^{\omega}$ and let $A$ be a compact segment in $E^{\omega}$. Consider the disjoint union $A \cup(C \times[0, \infty))$ of $A$ and the product space $C \times[0, \infty)$. Let $h$ be a one-to-one mapping from $A \cup(C \times[0, \infty))$ onto a continuum in $E^{\omega}$ such that the following properties are satisfied.

(1) $h(z)=z$ if $z \in A$.

(2) $h(z, 0)=z$ for all $z \in C$.

(3) $h \mid C \times[0, \infty)$ is a homeomorphism.

(4) $\lim _{t \rightarrow \infty} \delta(h(C \times\{t\}))=0$.

(5) For each $z \in C, h(\{z\} \times[0, \infty)) \cup A$ is homeomorphic to the closure of the curve in the plane $E^{2}$ whose equation is $y=\sin \pi / x$ for $0<x \leqslant 1$.

Received by the editors April 1, 1982.

1980 Mathematics Subject Classification. Primary 54C15, 54C55, 54F40.

Key words and phrases. Absolute (neighborhood) retract, absolute fixed point set, absolute approximate retract, contraction.

'The research for this article was supported in part by NSERC (Canada) Grant A8205. 
The image of $A \cup(C \times[0, \infty))$ under $h$ is defined to be the cap of $C$ and $A$, denoted by cap $C A$. We shall regard cap $C A$ as the set $A \cup(C \times[0, \infty))$ together with an assigned metric which makes the function $h$ an isometry.

Let $B$ be a subset of a space $X$. Then $B$ is called a fixed point set of $X$ if there is a self-mapping of $X$ whose fixed point set is $B$. We call $B$ an approximate retract of $X$ if for every neighborhood $U$ of $B$ in $X$ there is a retract $R$ of $X$ such that $B \subset R \subset U$. A compactum $X$ is an $A F S$-space ( $A A R$-space) if whenever $X$ is embedded as a subset of a compactum $Z$, then $X$ is a fixed point set (an approximate retract) of $Z$. It has been shown that both of these classes of spaces are properly contained in the class of contractible and locally contractible compacta $[3,4]$.

\section{Neighborhood contractible spaces.}

3.1. Definitions. (1) A space $X$ is neighborhood contractible at a subset $A$ if every neighborhood $U$ of $A$ in $X$ contains a neighborhood $V$ of $A$ in $X$ such that $V$ is contractible in $U$.

(2) A compactum $X$ is neighborhood contractible (or an NC-space) if $X$ is neighborhood contractible at each of its nonempty closed contractible subsets.

\subsection{Proposition. Every neighborhood retract of an NC-space is an NC-space.}

Proof. Suppose $X$ is a neighborhood retract of an NC-space $Z$. Then there is an open set $Y$ containing $X$ and a retraction $r: Y \rightarrow X$. Let $K$ be a nonempty closed contractible subset of $X$ and let $U$ be an open set in $X$ containing $K$. Since $M=r^{-1}(U)$ is an open subset of $Z$ containing $K$, there is a neighborhood $W$ of $K$ in $Z$ such that $K \subset W \subset M$ and $W$ is contractible in $M$. Let $H: W \times I \rightarrow M$ be a contraction of $W$ in $M$. Let $V=W \cap U$ and define $F: V \times I \rightarrow U$ by $F(x, t)=$ $r H(x, t)$ for $(x, t) \in V \times I$. Then $V$ is a neighborhood of $K$ in $X$ and $F$ is a contraction of $V$ in $U$ as required.

Since each factor in a cartesian product space is embedded as a retract of that space, we obtain the following corollary to Proposition 3.2.

3.3. COROLlaRY. If the cartesian product space $\prod_{1=1}^{\infty} X_{i}$ is an NC-space, then every $X$, is an NC-space.

\subsection{THEOREM. Every ANR-space is an NC-space.}

Proof. Let $X$ be an ANR-space and suppose $A$ is a nonempty closed contractible subset of $X$. Let $U$ be an open subset of $X$ which contains $A$ and let $K$ be a compact neighborhood of $A$ lying in $U$. Since $A$ is contractible, there is a contraction $F$ : $A \times I \rightarrow A$ which deforms $A$ to a point $p$. Define a mapping $G:(K \times\{0\}) \cup(A \times$ I) $\cup(K \times\{1\}) \rightarrow U$ by

$$
G(x, t)= \begin{cases}x & \text { if } x \in K \text { and } t=0 \\ F(x, t) & \text { if }(x, t) \in A \times I, \\ p & \text { if } x \in K \text { and } t=1\end{cases}
$$

Since $U$ is an absolute neighborhood retract for the class of metric spaces [1, p. 96], there is a neighborhood $W$ of $(K \times\{0\}) \cup(A \times I) \cup(K \times\{1\})$ lying in $X \times I$ 
and an extension $H: W \rightarrow U$ of $G$. It is easy to show that there is a neighborhood $V$ of $A$ in $X$ such that $V \subset K$ and $V \times I \subset W$ (see [2,p. 86]). Then the restriction map $H \mid V \times I: V \times I \rightarrow U$ is a contraction of $V$ in $U$ which deforms $V$ to the point $p$. Therefore $X$ is an NC-space as required.

Since neighborhood contractibility implies local contractibility and every finitedimensional locally contractible compactum is an ANR-space [1, p. 122], we obtain the following corollary to Theorem 3.4 .

3.5. COROllary. For a finite-dimensional compactum $X$, the following three statements are equivalent.

(1) $X$ is neighborhood contractible.

(2) $X$ is locally contractible.

(3) $X$ is an $A N R$-space.

In the proof of Theorem 3.6 (Theorem 3.7) we rely heavily on both the statement and the initial constructions used in the proof of Theorem 2 in [3] (Theorem 1 in [4]).

\subsection{THEOREM. Every AFS-space is an NC-space.}

Proof. Let $X$ be an AFS-space and suppose that $X$ is not an NC-space. Then there is a nonempty closed contractible subset $K$ in $X$ and a neighborhood $U$ of $K$ in $X$ which contains a decreasing sequence $V_{1}, V_{2}, \ldots$ of compact neighborhoods of $K$ such that $\lim _{i \rightarrow \infty} V_{i}=K$ and no $V_{i}$ is contractible in $U$. Let $A_{i}, i=1,2, \ldots$, denote the subinterval of the closed unit interval $I=[0,1]$ defined by

$$
A_{i}=[1 / 2 i, 1 /(2 i-1)] \text {. }
$$

Consider a sequence of disjoint continua $Y_{1}, Y_{2}, \ldots$ with $\lim _{i \rightarrow \infty} Y_{i}=\lim _{i \rightarrow \infty} V_{i}=K$ obtained by first taking the disjoint union $\cup_{i=1}^{\infty} V_{i}$ and then letting $Y_{i}=\operatorname{cap} V_{i} A_{i}$ for $i=1,2, \ldots$. Let $Y=I \cup \cup_{i=1}^{\infty} Y_{i}$. Form the compactum $Z$ obtained by taking the disjoint union $X \cup Y$ and then identifying $V_{i} \times\{0\}$ in $Y$ with $V_{i}$ in $X$ for $i=1,2, \ldots$, and identifying the point 0 in $I$ with a point $p$ in $K$.

Now, since $X$ is an AFS-space, there is a map $f: Z \rightarrow Z$ whose fixed point set is $X$. Let $Q=\cup\left\{Y_{i} \mid f\left(A_{i}\right) \cap(0,1]=\varnothing\right\}$. Then the arguments used in the proof of Theorem 2 in [3] show that all but finitely many of the $Y_{i}$ are subsets of $Q$ and there is a retraction $r: f(X \cup Q) \rightarrow X$. Since $\lim _{i \rightarrow \infty} Y_{i}=K$, there is some $Y_{k}$ such that $r f\left(Y_{k}\right) \subset U$. Let $a_{k}$ denote the midpoint of $A_{k}$. Then, since $X$ is locally contractible [3, Theorem 2], there is a neighborhood $W$ of $r f\left(a_{k}\right)$ in $U$ and a deformation $\beta$ : $W \times I \rightarrow U$ which deforms $W$ to a point. Since $r f$ is continuous at $a_{k}$, there is a neighborhood $N$ of $a_{k}$ in $Y_{k}$ such that $r f(N) \subset W$. Then, for some $s$ in $[0, \infty)$, we have $V_{k} \times\{s\} \subset N$. Let $\alpha: V_{k} \times I \rightarrow Y_{k}-A_{k}$ denote a deformation which deforms $V_{k}=V_{k} \times\{0\}$ onto $V_{k} \times\{s\}$. Define a homotopy $H: V_{k} \times I \rightarrow U$ by

$$
H(x, t)= \begin{cases}r f \alpha(x, 2 t) & \text { if } 0 \leqslant t \leqslant \frac{1}{2}, \\ \beta(r f \alpha(x, 1), 2 t-1) & \text { if } \frac{1}{2} \leqslant t \leqslant 1\end{cases}
$$

It is easy to check that $H$ is a deformation which deforms $V_{k}$ to a point in $U$. This contradiction shows that $X$ must be an NC-space. 


\subsection{THEOREM. Every AAR-space is an NC-space.}

Proof. Let $X$ be an AAR-space and suppose that $X$ is not an NC-space. Then let $K, U, V_{i}, A_{i}, Y_{i}, i=1,2, \ldots$, be as in the proof of Theorem 3.6. Form the continuum $M$ obtained by taking the disjoint union of $X$ and $\cup_{i=1}^{\infty} Y_{i}$, and then identifying $V_{i}$ in $X$ with $V_{i} \times\{0\}$ in $Y_{i}$ for $i=1,2, \ldots$ A construction similar to that found in [4, p. 298 ] can be used to attach an infinite ray $A$ to $M$ to obtain a new continuum $Z$. The continuum $Z=M \cup A$ may be regarded as a subspace of $E^{\omega}$ where $A$ is a homeomorph of $[0, \infty)$ which is disjoint from $M$ and "converges" to a subcontinuum of $M$ which contains $\cup_{i=1}^{\infty} A_{i}$. In particular, every open set in $Z$ containing a point belonging to some $A_{i}$ must contain a subinterval of $A$.

Let $W=Z-C$ where $C$ is a compact neighborhood of $A_{1}$ in $Z$ such that $C \cap\left(\cup_{i=2}^{\infty} Y_{i} \cup X\right)=\varnothing$. Since $X$ is an AAR-space, there is a retraction $f: Z \rightarrow R$ where $R$ is a retract of $Z$ such that $X \subset R \subset W$. A simple argument which can be found in the proof of Theorem 1 in [4] shows that there is a retraction $r: R \rightarrow X$ such that $r f\left(Y_{i}\right) \subset U$ for all but finitely many of the $Y_{i}$. Since the AAR-space $X$ is locally contractible [4, Theorem 1], the remaining argument needed to complete the proof is identical to that used in the proof of Theorem 3.6.

It has been shown in Corollary 3.5 that, for finite-dimensional compacta, the concepts of neighborhood contractible and locally contractible coincide. We now show that a well-known example due to Borsuk [1, p. 126] is, in fact, an example of a contractible and locally contractible compactum which is not neighborhood contractible.

3.8. EXAMPLE. Consider the following subsets of the Hilbert cube $Q^{\omega}$ (for notation see $[1$, p. 10]):

$$
\begin{aligned}
& X_{0}=\left\{x=\left\{x_{i}\right\} \mid x_{1}=0\right\}, \\
& B_{k}=\left\{x=\left\{x_{i}\right\} \mid \frac{1}{k+1} \leqslant x_{1} \leqslant \frac{1}{k} \text { and } x_{i}=0 \text { for } i>k\right\} \text { for } k=1,2, \ldots
\end{aligned}
$$

The boundary $\mathrm{Bd} B_{k}$ of $B_{k}$ is a $(k-1)$-sphere which we shall denote by $X_{k}$ for $k=1,2, \ldots$ Let $X=X_{0} \cup \cup_{k=1}^{\infty} X_{k}$. Then, if $Y$ denotes the cone over $X$ with vertex $p, Y$ is a contractible and locally contractible compactum which is not an ANR-space $[1$, p. 126]. We now show that $Y$ is not neighborhood contractible.

To facilitate notation, we shall identify $X$ with $X \times\{0\}$ in $Y=\operatorname{Cone}(X)$. Then $X_{0}$ is a contractible subset of $Y$ which is, in fact, homeomorphic to the Hilbert cube $Q^{\omega}$. Consider the neighborhood $Y-\{p\}$ of $X_{0}$ in $Y$. Let $V$ be any neighborhood of $X_{0}$ in $Y-\{p\}$. Then $V$ contains infinitely many of the spheres $X_{k}, k=1,2, \ldots$. Since no $X_{k}, k=1,2, \ldots$, is contractible in $Y-\{p\}$, it follows that ${ }^{\cdot} Y$ is not neighborhood contractible at $X_{0}$. Consequently, $Y$ is not an NC-space.

Since the class of ANR-spaces is contained in the class of NC-spaces which, in turn, is properly contained in the class of locally contractible compacta, it seems appropriate to pose the following question.

3.9. QUESTION. Does the class of NC-spaces coincide with the class of ANR-spaces?

We remark that the results of this paper (see the last sentence in \$2) show that every AFS-space (AAR-space) is a contractible NC-space. Since every contractible 
ANR-space is an AR-space [1,p. 101], a positive answer to Question 3.9 would show that the concepts of an AFS-space, AAR-space and AR-space are equivalent.

\section{REFERENCES}

1. K. Borsuk, Theory of retracts, Monografie Mat., vol. 44, PWN, Warsaw, 1967.

2. W. Hurewicz and H. Wallman. Dimension theory. Princeton Univ. Press, Princeton, N. J., 1941.

3. J. R. Martin, Absolute fixed point sets and AR-spaces, Fund. Math. 112 (1981), 159-164.

4. Absolute approximate retracts and AR-spaces, Canad. J. Math. 33 (1981), 297-301.

Department of Mathematics. University of Saskatchewan, Saskatoon, Saskatchewan, Canada S7N 0 W0 